\title{
POSTER: Performance Evaluation of Key Management Schemes for Wireless Legacy Smart Grid Environments
}

\author{
Mumin Cebe and Kemal Akkaya \\ Dept. of Electrical and Computer Engineering, Florida International University \\ Miami, FL, USA 33174 \\ \{mcebe,kakkaya\}@fiu.edu
}

\begin{abstract}
With the increasing digitization of different components of Smart Grid, there is an ongoing effort to design secure protocols and deploy them for different applications. A major need along with these efforts is to deal with key management for a large number of devices. While key management can be easily addressed by transferring the existing protocols to Smart Grid domain, this is not an easy task as one needs to deal with the limitations of the current communication infrastructures and resource-constrained devices. As the utilities rightly requests to build the new systems on top of the legacy systems with limited investment, the research community needs to re-think the adaptation of the existing security approaches to such non-traditional environments. This poster aims to tackle one of these problems, namely, symmetric key management in a severely constrained wireless communication environment. Assuming a legacy radio communication infrastructure with bandwidths in the order of kilobits, the objective is to evaluate the feasibility and performance of the existing sophisticated key management protocols. We developed a realistic ns-3 environment and analyze the delay overhead via simulations.
\end{abstract}

\section{CCS CONCEPTS}

- Security and privacy $\rightarrow$ Mobile and wireless security;

\section{KEYWORDS}

smart grid, legacy wireless infrastructure, key management, efficiency.

\section{ACM Reference format:}

Mumin Cebe and Kemal Akkaya. 2019. POSTER: Performance Evaluation of Key Management Schemes for Wireless Legacy Smart Grid Environments. In Proceedings of WiSec '19: ACM Conference on Security and Privacy in Wireless and Mobile Networks, Miami, FL, USA, May 15-17, 2019 (WiSec '19), 2 pages.

https://doi.org/10.1145/3317549.3326318

\section{INTRODUCTION}

As the communication within the Smart Grid needs to be secure, key exchange becomes a challenge due to its overhead on some of the legacy communication infrastructures that are part of the power

Permission to make digital or hard copies of part or all of this work for personal or classroom use is granted without fee provided that copies are not made or distributed for profit or commercial advantage and that copies bear this notice and the full citation on the first page. Copyrights for third-party components of this work must be honored.

For all other uses, contact the owner/author(s).

WiSec '19, May 15-17, 2019, Miami, FL, USA

(C) 2019 Copyright held by the owner/author(s)

ACM ISBN 978-1-4503-6726-4/19/05 .. \$15.00

https://doi.org/10.1145/3317549.3326318 grid [7]. Specifically, in such legacy communication infrastructures, intelligent electronic devices (IEDs) or distributed energy resources (DERs) are connected to the control center via a low-bandwidth point to multi-point radio communication links as shown in Fig. 1.

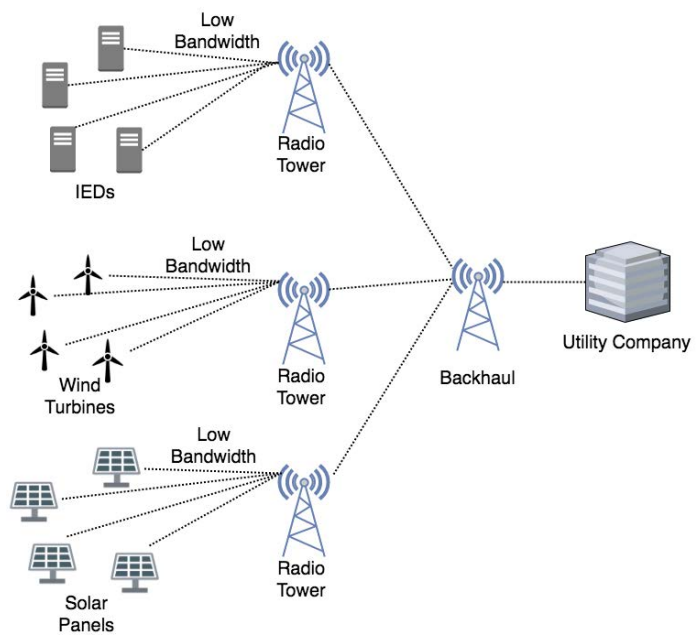

Figure 1: An overview of a sample legacy infrastructure.

Due to the communication overhead of key exchange in such lowbandwidth links (which is also used for uplink and downlink data transfers), a naive solution utilities follow is to use the same key for a long period of time. However, this long-period key has very lowsecurity features as compromising one key means compromising forward secrecy during that period.

Furthermore, using existing key management protocols that are designed for resource-rich wired networks is not feasible as they will congest the links, hindering the actual data transfer and eventually causing longer delays which may not be acceptable for power flow control. In general, effective security mechanisms for Smart Grid must guarantee the security of any applications running on it without compromising their general performance through minimal overhead and interoperability capabilities with the existing legacy systems. When offering security and key management solutions to utilities, the assumptions that they will update their existing systems often results in failures and thus necessitates in advance assessments.

In this study, we build a realistic simulation infrastructure to evaluate the latency overhead of different existing and emerging key-exchange schemes during the collection of data from IEDs and DERs. We use ns-3 simulator [8] to model the LoRa [1] characteristics as a low-bandwidth communication infrastructure (i.e., order 
of kbits) to mimic the underlying legacy communication infrastructure that is currently being used in many utilities in the US. We then implement and evaluate well-known key exchange mechanisms that are currently in use for Internet applications.

\section{PRELIMINARIES}

Well-known key exchange protocols did not consider much about the efficiency or overhead. For instance, IKE version v1 (as part of IPSec) needs up to 4.5 round-trip time (RTT) [5], the improved version of it (IKEv2) requires 3 RTT [6] and datagram transport layer security (DTLS) requires 3 RTT while using UDP instead of TCP [9]. Reducing RTT overhead of key exchange while providing security guarantees is critical considering the low-bandwidth communication infrastructure of smart grid. This particular goal is also becoming a major interest of in industry. For instance, Noise protocol [3] which is used by Whatsapp and Lightning Network requires only one RTT for the key exchange, thereby minimizing latency. A prominent example for minimizing latency is introduced by Google via QUIC protocol which allows the parties to accomplish key-exchange in zero RTT [4]. At a high level overview, QUIC works as follows: If a client is interacting with a server for the first time, it sends a CONFIG_REQ message and downloads a server configuration file over 1-RTT. This contains a signed Diffie-Hellman (DH) component [2] which will be used by the client to derive a secret key over 0-RTT.

\section{EVALUATIONS}

\subsection{Experimental Setup}

To assess the performance of the different key-exchange mechanisms, we created a legacy environment by utilizing LoRa in ns-3. LoRa is a technology which is designed to transmit information at long distances (e.g., $2 \mathrm{~km}$ in urban and $10 \mathrm{~km}$ in rural) by adjusting its transmission bit-rate in the order of kbits [1]. This mimics the available resources currently in use by some utilities. In our simulated network, we consider Class A LoRa scheme which enables access the wireless channel for uplink transmissions to the gateway following an ALOHA scheme. We simulated the characteristics of LoRa for a typical $1 \mathrm{~km}$ range in an urban environment and a typical $10 \mathrm{~km}$ range in a rural environment. For virtualization of the IEDs and the gateway which collects the data, we setup Linux containers [10] and installed wolfssl [11] library to establish a secure communication between IEDs and utility company through LoRa.

\subsection{Experiment Results}

We conducted two different experiments to assess the performance of different key-exchange mechanisms for different LoRa settings according to urban and rural communication environment.

The results which are shown in Fig. 2 indicate that 0-RTT approach (QUIC) significantly reduces the data submission time compared to the other approaches due to decreased number of messages during the handshake. Even with respect to DTLS, which is touted as one of the most efficient methods for secure transport layer in low-bandwidth environments, 0-RTT approach reduced the completion time in approximately 2 orders of magnitude for the urban and 8 orders of magnitude for the rural environment. However, it is interesting to observe that the performance gap for DTLS in urban

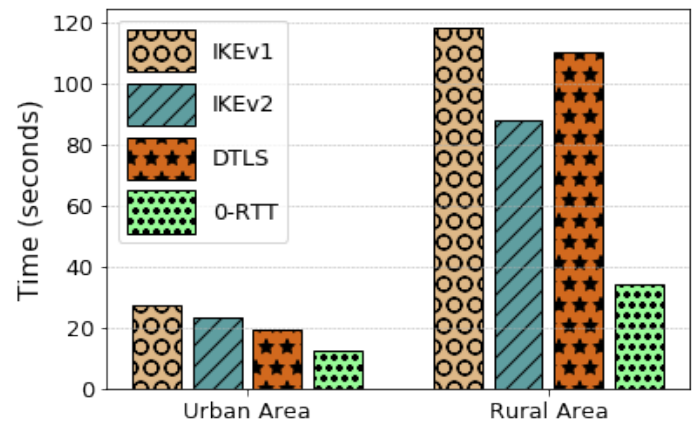

Figure 2: The power reading submission time for different environmental settings.

environments widens significantly. Despite the use of TCP with additional packet overhead, 0-RTT performs much better due to TCP's congestion control features. Hence, the results defeats the existing assumptions that UDP should be used to reduce overhead. Due to increased congestion in low-badwidth links, TCP is helpful to exploit its congestion handling features.

\section{CONCLUSION}

The overall purpose of this study was assessing the overhead of key-exchange mechanisms in the low-bandwidth communication environments for Smart Grid. To do so, we built a simulation environment by using ns-3 to mimic the communication characteristics of LoRaWan. The results showed the superior performance of the 0 RTT comparing to the other key-exchange mechanisms, indicating that TCP can still be a viable option in such environments.

\section{ACKNOWLEDGMENTS}

This work is supported by DOE Award Number DE-OE0000779 .

\section{REFERENCES}

[1] Ferran Adelantado, Xavier Vilajosana, Pere Tuset-Peiro, Borja Martinez, Joan Melia-Segui, and Thomas Watteyne. 2017. Understanding the limits of LoRaWAN. IEEE Communications magazine 55, 9 (2017), 34-40.

[2] Whitfield Diffie and Martin Hellman. 1976. New directions in cryptography. IEEE transactions on Information Theory 22, 6 (1976), 644-654.

[3] Facebook. 2016. Noise Protocol Framework. Release. (2016). http://noiseprotocol. org/

[4] Google. 2016. QUIC Proocol. Release 3.24.1. (2016). https://www.chromium.org/ quic

[5] Paul Hoffman. 2005. Algorithms for internet key exchange version 1 (ikev1). (2005).

[6] Charlie Kaufman, Paul Hoffman, Yoav Nir, Pasi Eronen, and Tero Kivinen. 2014. Internet key exchange protocol version 2 (IKEv2). Technical Report.

[7] Xuelian Long, David Tipper, and Yi Qian. 2013. An advanced key management scheme for secure smart grid communications. In 2013 IEEE International Conference on Smart Grid Communications (SmartGridComm). IEEE, 504-509.

[8] ns 3. 2016. ns-3: Network Simulator 3. Release 3.24.1. (2016). http://www.nsnam. org/

[9] Eric Rescorla and Nagendra Modadugu. 2012. RFC 6347: Datagram transport layer security version 1.2. Internet Engineering Task Force 13 (2012), 101.

[10] Rami Rosen. 2014. Linux containers and the future cloud. Linux f 240, 4 (2014), 86-95.

[11] SSL WolfSSL-Embedded. [n. d.]. Library for Applications, Devices, IoT, and the Cloud. ([n. d.]) 